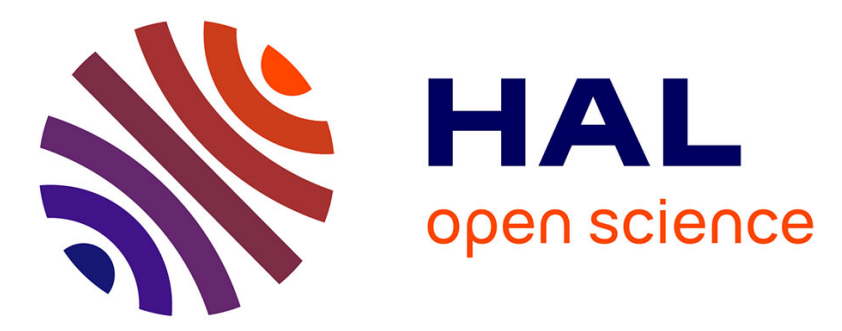

\title{
Development and validation of the numerical model of an innovative PCM based thermal storage system
}

\author{
Letizia Roccamena, Mohamed El Mankibi, Nikolaos Stathopoulos
}

\section{To cite this version:}

Letizia Roccamena, Mohamed El Mankibi, Nikolaos Stathopoulos. Development and validation of the numerical model of an innovative PCM based thermal storage system. Journal of Energy Storage, 2019, 24, pp.100740. 10.1016/j.est.2019.04.014 . hal-03116406

\section{HAL Id: hal-03116406 \\ https://hal.science/hal-03116406}

Submitted on 22 Oct 2021

HAL is a multi-disciplinary open access archive for the deposit and dissemination of scientific research documents, whether they are published or not. The documents may come from teaching and research institutions in France or abroad, or from public or private research centers.
L'archive ouverte pluridisciplinaire HAL, est destinée au dépôt et à la diffusion de documents scientifiques de niveau recherche, publiés ou non, émanant des établissements d'enseignement et de recherche français ou étrangers, des laboratoires publics ou privés.

\section{다)(1) $(5$}

Distributed under a Creative Commons Attribution - NonCommercial| 4.0 International 
1 Development and validation of the numerical model of an innovative PCM

2 based thermal storage system

3 Letizia Roccamena ${ }^{a^{*}}$, Mohamed El Mankibi ${ }^{a}$, Nikolaos Stathopoulos $^{b}$

4

5

${ }^{a}$ Laboratory of Tribology and Systems Dynamics (LTDS), Ecole Nationale des Travaux Publics de l'État (ENTPE), 3 Rue Maurice Audin, Vaulx-en-Velin, France

${ }^{\mathrm{b}}$ Department of Architecture and Civil Engineering, Sven Hultins gata 6, Chalmers University of Technology, Division of Building Technology, Gothenburg, SE-412 96, Sweden

*Corresponding author. E-mail address: letizia.roccamena@entpe.fr

\section{ABSTRACT}

Because of the high costs of experimental tests in the real conditions of buildings, numerical simulation, developed analytical methods and different modelling studies are needed to predict the behaviour and results of phase change materials (PCMs) usage in buildings in order to optimize the thermal energy storage techniques and to make them more efficient and cost-effective. The aim of this study is to develop a numerical model reproducing the behaviour of an innovative water-PCM heat exchanger for cooling purposes particularly created for HIKARI, the first positive energy, mixed use district in France. Once numerically calibrated and experimentally validated, this model was used to optimize the system's technology applying Genetic Algorithms methods. The model presented in this article was developed based on the heat balance approach and solved using the finite difference method. It was validated both numerically, using a Computational Fluid Dynamics model and experimentally using both the results of an innovative experimental prototype designed and constructed in laboratory conditions and HIKARI in situ monitoring results. The Normalized Mean Bias Error and the Coefficient of Variation of the Root Mean Squared Error, used to analyse the validation results, show that the choice of the heat balance approach provided a valid model able to reproduce the PCM-water heat exchange with high accuracy.

\section{KEYWORDS}


31 Thermal energy storage (TES) is a technology that can enable greater and more efficient use of renewable energy sources by matching the energy supply with the demand, as it consists of stocking thermal energy by heating a material (the "Heat Storage Medium") capable to release the stored thermal energy at a later time. In this way, this technology can help balance energy consumption and reduce peak demand, $\mathrm{CO}_{2}$ emissions and costs, while increasing overall efficiency of energy systems [1-4]. In view of this, TES is considered one of the most promising technologies, as it can play a key role in energy efficient buildings by stocking thermal energy in order to reduce indoor air temperature fluctuation or reinstituting it to the system (building, district, and town) at a later period with an hourly, daily or even seasonal time lag [5-8]. The current study aims to design, model and numerically and experimentally validate a water /PCM heat exchanger with TES purposes [9]. This system is part of the multi-energy production and storage systems of HIKARI project (Figure 1), a positive energy district located in Lyon consisting of three buildings, combining commercial, residential and office usage [10].

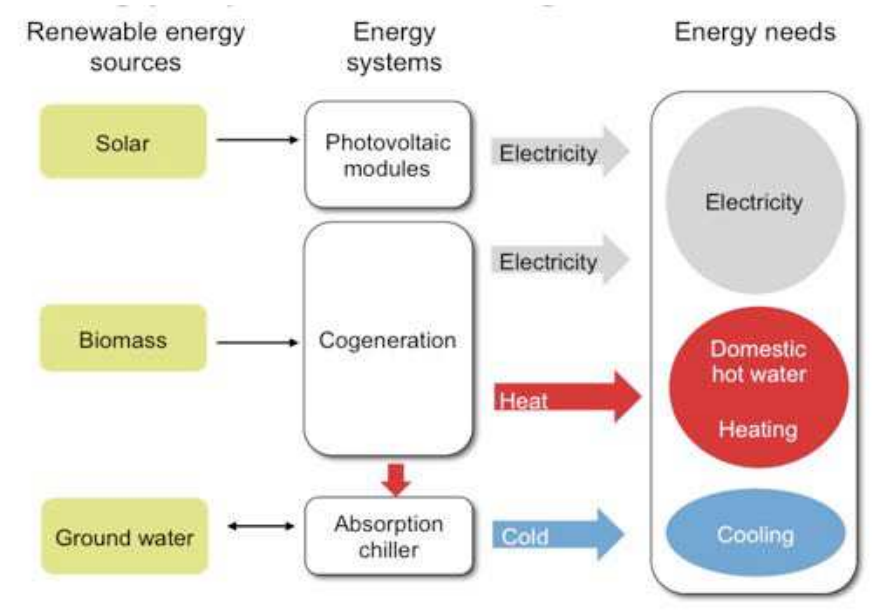

The scheme of the HVAC technology of the HIKARI district is shown in Figure 2. The cooling system includes an absorption chiller, an adiabatic dry cooler, a vapor-compression refrigeration system and a storage system, while the heating system includes a cogeneration unit feed with rapeseed oil, a gas boiler (that is connected to the absorption chiller) and a storage system. The PCM based thermal storage system at low temperatures (indicated in the green circle) is connected to the HIKARI's absorption chiller $(46 \mathrm{~kW})$ that provides the water during the charging and discharging phases. This cold water storage system is used in order to improve the performance of the absorption chiller. 


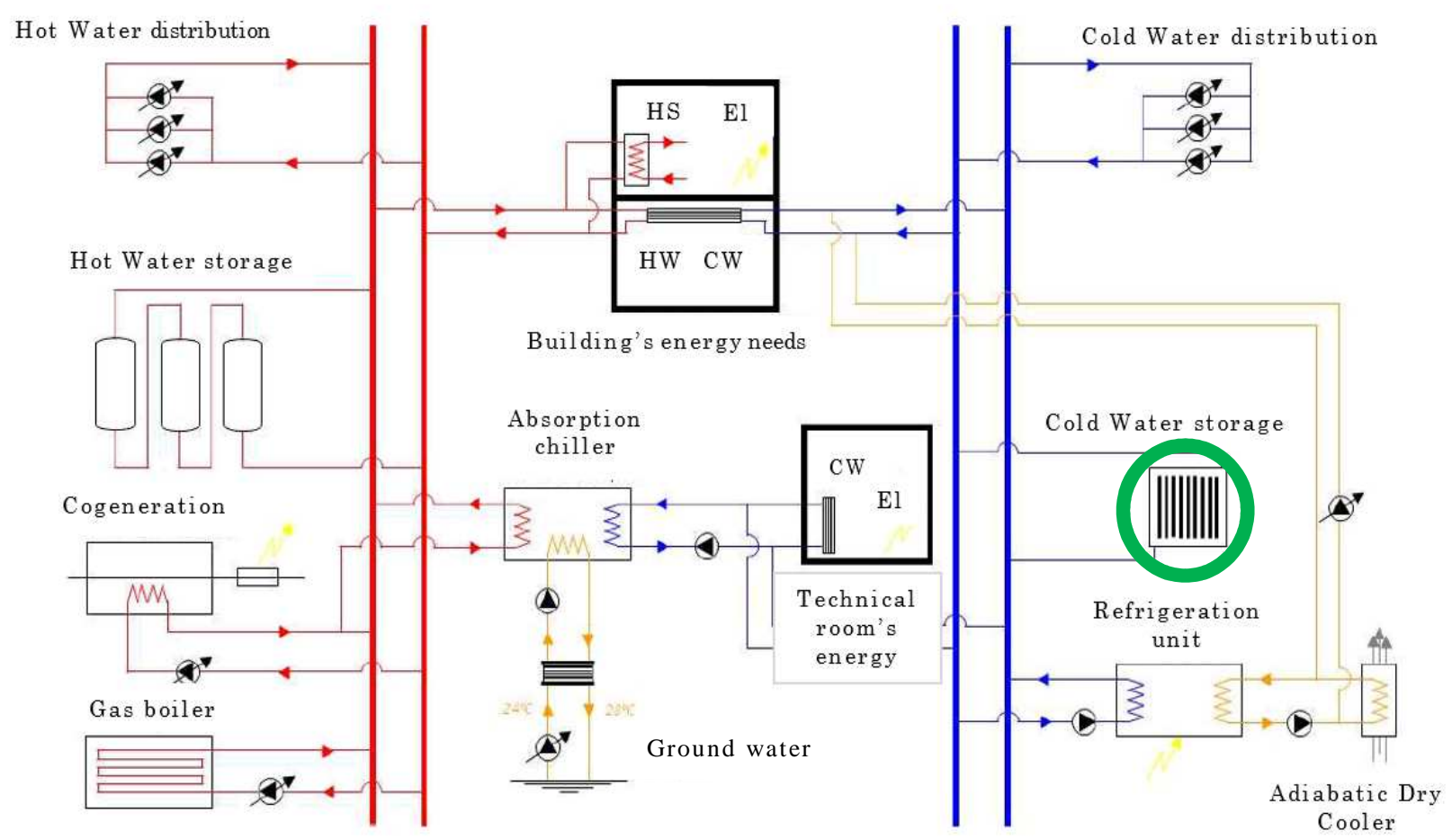

Figure 2 - Scheme of the HVAC technology of HIKARI. The reference thermal energy storage system is indicated in the green circle.

68

The positive effect of PCMs integration on the performance of air conditioning systems has already been tested in different studies [11-13]. Nevertheless, the challenges linked to the big dimensions of the HIKARI district and the presence of its multi-source energy architecture, made the technology of this thermal storage system particularly complex.

Concerning its technology, the water-PCM heat exchanger consists of $27 \mathrm{~m}^{3}$ of phase change material (PCM) (subject to a phase change between 9 and $11^{\circ} \mathrm{C}$ ) processed into a gelatinous form and enclosed in impermeable cylindrical stick packages (Figure 3.a). Each cylindrical package encloses $100 \mathrm{~g}$ of a PCM, the JX Nippon Oil branded paraffin Ecojoule ${ }^{\circledR}$, able to store and to release approximately $154 \mathrm{~kJ} / \mathrm{kg}$. The sticks are inserted in plastic cases (Figure 3.b), which are subsequently inserted in four insulated thermal storage tanks (Figure 3.c) filled with water. The total thermal energy storage capacity of the system is $3000 \mathrm{MJ}$.
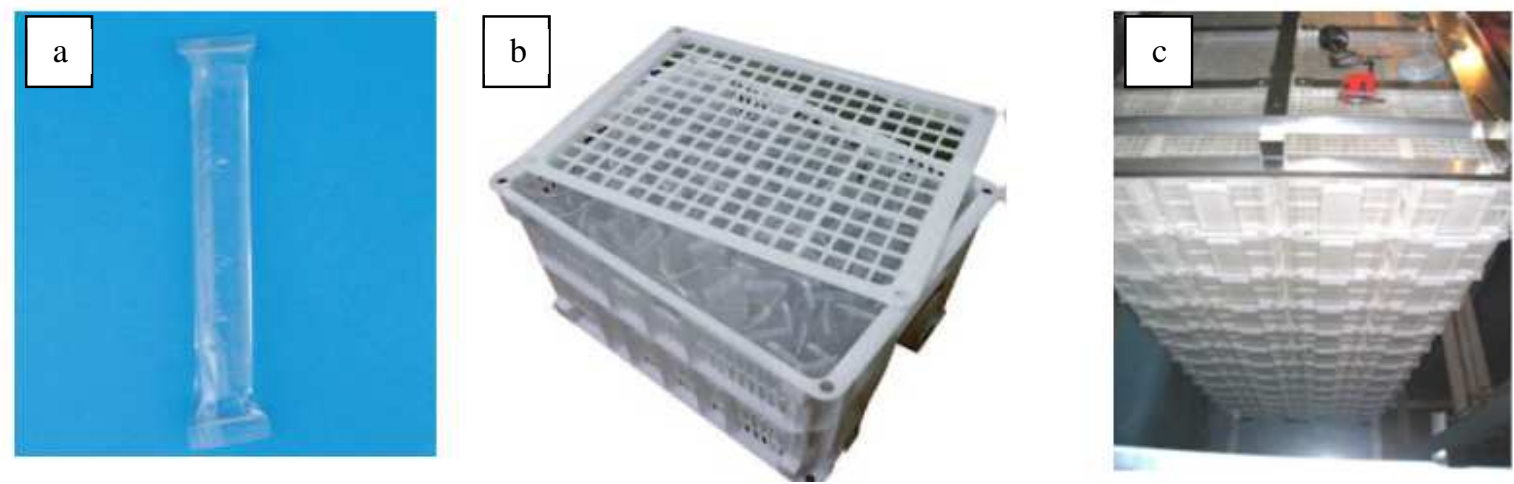

Figure 3 - (a) Latent Heat Thermal Storage material package (gel pack), (b) plastic case filled with gel packs and (c) typical installation of the plastic cases into the isolated tank. 
Water circulates into this tank as the heat transfer fluid, entering in the system from tubes situated on the top of the tank, passing from small openings with a flow rate of $50 \mathrm{~g} / \mathrm{s}$. For all the tanks, 180 opening can be counted (each opening is positioned in front of a single plastic case). The plastic cases have large apertures so as not to impede the water flow that runs into the tank when they are stacked in it. After passing through the gel sticks layer, the water exits the system passing through openings present in other tubes located at the bottom of the tanks (Figure 4).

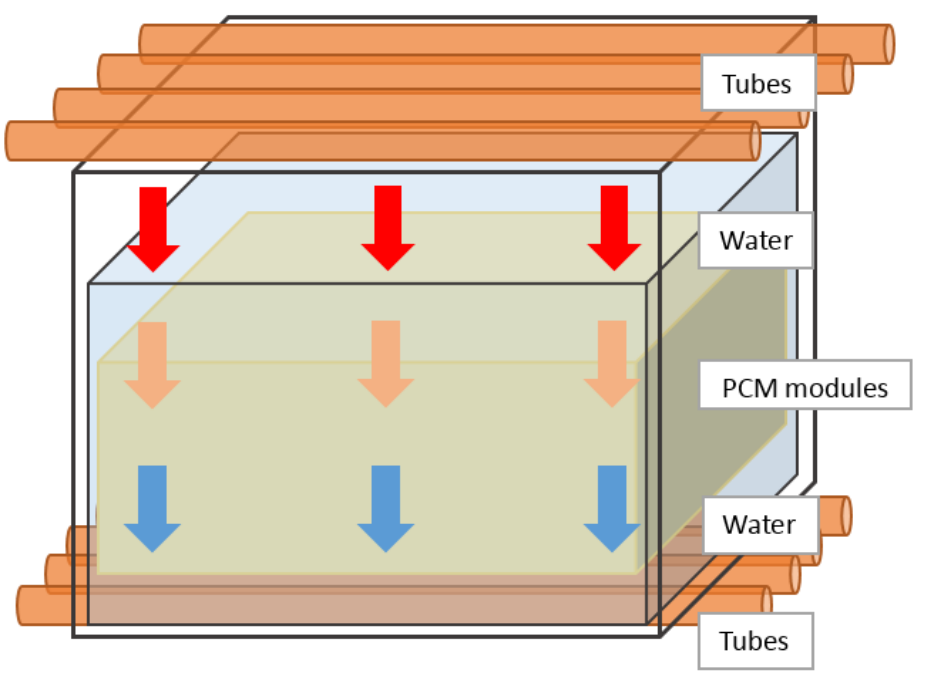

Figure 4 - Scheme of one of the four tanks composing the system.

Once the temperature of the water into the four tanks is $6^{\circ} \mathrm{C}$, the system is considered " $100 \%$ charged", and the water that it contains is sent to the absorption chiller, reducing the difference between the chiller's cold and hot inlet water temperature. After the heat exchange, the water flow is redirected to the storage tank continuing this cycle until the tank's temperature reaches $10.5^{\circ} \mathrm{C}$. At that point, the system is considered "discharged", remaining unavailable until it is charged again.

\section{NUMERICAL MODELLING}

\subsection{The heat balance approach}

Contrary to experimental studies, numerical simulations can help to analyse physical phenomena quickly and cost-effectively [14]. Furthermore, validated models can be used in parametric studies having a greater versatility compared to experimental prototypes.

Fundamentally, the developed models are based on the formulation of a series of energy related equations and their solutions using analytical and numerical methods. Because of the complexity linked to the calculation of the moving boundary of phases, numerical methods are more frequently used for phase change problems [15-17]. In order to obtain a dynamic model able to reproduce the behaviour of our reference technology, the heat balance approach 
was chosen. It is based on the division of the analysed system into a defined number of volume elements (it is a fixed grid method) and the subsequent application of the energy balance equations for each of them. The heat balance equations are formed for every volume element and solved at each time step in order to calculate the temperature evolution at the considered nodes.

This approach assumes that the temperature value is the same for each volume element. When the system is composed of two or more materials (as in our case with water and PCM) different layers are considered, in order to better study the behaviour of each material.

In this study, the first step consisted in analysing all the relevant energy flows present in the system, in order to write the energy balance equations, considering conduction, convection and advection transfers. Once the energy balance equations were written, the finite difference method was employed, in order to approximate the partial differential equations. Finally, the equations were solved at each time step and for each volume through the software MATLAB Simulink.

This method was chosen as it was previously used and provided good results for similar configurations $[18,19]$.

\subsection{Selection of the development environment}

As the HIKARI's HVAC model was created under MATLAB-Simulink environment, this software was selected for the development of the model reproducing the HIKARI's cold storage system energy behaviour. Also, the software offers the possibility of fast calculations and modification of several parameters for the subsequent optimization phase [20].

\subsection{The apparent heat capacity method}

The heat capacity and enthalpy methods are often used to model the PCM thermal behaviour in buildings [21, 22]. In this case, the problem of the phase change of the heat storage medium was resolved using the "apparent heat capacity method", that consists in representing the phase change through an apparent increase of the material's heat capacity value for a certain temperature range; this increase represents the corresponding latent heat absorption/release [23-24].

In case of uniform process, we can define the apparent heat capacity $(C p)$ as:

(1)

$$
C_{p, \text { app }}=\left\{\begin{array}{c}
C_{p, s}\left(\text { for the solid phase, where } T<T_{s}\right) \\
C_{p}(T)\left(\text { for the phase change, where } T_{s}<T<T_{l}\right) \\
C_{p, l}\left(\text { for the liquid phase, where } T>T_{l}\right)
\end{array}\right.
$$

In order to obtain the $\mathrm{C}_{\mathrm{p}}$ values of the Ecojoule ${ }^{\circledR} \mathrm{PCM}$, a Differential Scanning Calorimetry (DSC) test was made. 
129 The heat exchange between the PCM and the water flow was modeled by reproducing the

130 thermal exchange between a single PCM gel stick and water.

131 As the thickness of the enclosing plastic film of the gel sticks is minor $(0.5 \mathrm{~mm})$, the thermal resistance of this layer is considered negligible. Thus, two layers were considered for the discretization: PCM and water. Each layer was then discretized lengthwise into $\mathrm{n}$ equal regions (nodes) of length $\mathrm{L} / \mathrm{n}$, where $\mathrm{L}$ is the total length of the PCM/water exchanger (length of the PCM stick). The PCM layer was further discretized crosswise in m nodes which correspond to $m$ concentric cylinders with a thickness of $r=R / m$ where $R$ is the radius of the gel stick contained in a cylinder of water ( $\mathrm{i}=\mathrm{w}$ node) (Figure 5.a). The discretization into multiple nodes offers the possibility of a more thorough treatment of the medium where the phase change occurs. Subscript letter $\mathrm{j}$ signifies the nodal lengthwise position for each layer ( $j=1$ to $n$ ) and the subscript $\mathrm{i}$ signifies the nodal crosswise position in the PCM layer ( $\mathrm{i}=1$ to $\mathrm{m})$. The thermal resistances (represented with black rectangles) are formulated between nodes (represented with red dots) (Figure 5.b).
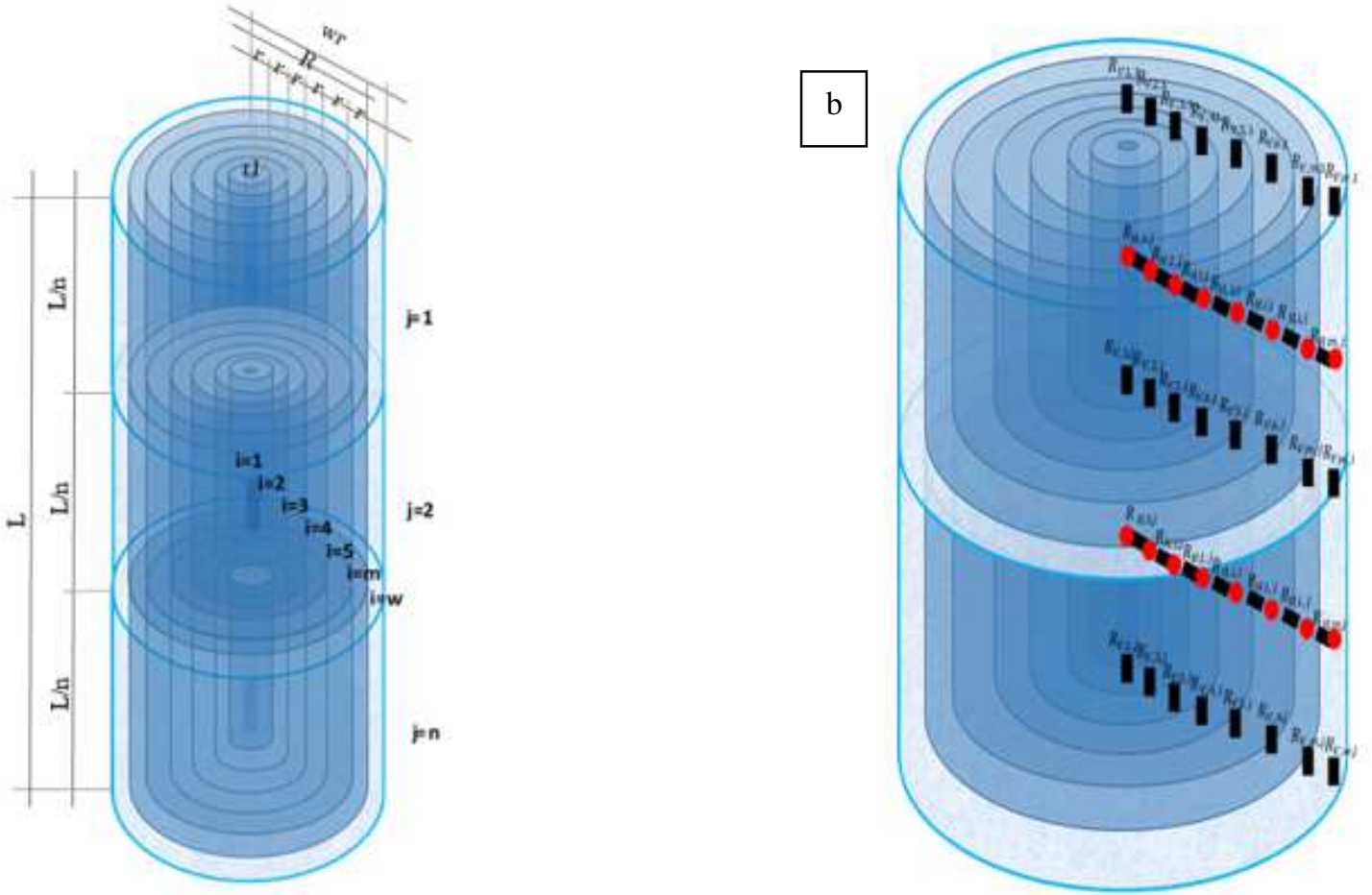

In order to write the energy balance equations we considered conduction in PCM, convection between water and PCM and advection in water as the possible transfer phenomena that could take place in each node, so the equations could be written as follows:

$$
\left\{\begin{array}{c}
\text { Temperature } \\
\text { change rate } \\
\text { in node }
\end{array}\right\}=\left\{\begin{array}{c}
\text { Conduction } \\
\text { transfers } \\
\text { in node }
\end{array}\right\}+\left\{\begin{array}{c}
\text { Convective } \\
\text { transfers } \\
\text { in node }
\end{array}\right\}+\left\{\begin{array}{c}
\text { Advection } \\
\text { transfers } \\
\text { in node }
\end{array}\right\}
$$


165 The energy balance equation was written for each node, taking into account that the heat

166 entering into that node is equal to the heat exiting that position.

167 The temperature change rate (or storage of heat in the node) can be expressed as follows:

168

169

170

171 Where:

$$
\left\{\begin{array}{c}
\text { Temperature } \\
\text { change rate } \\
\text { in node }
\end{array}\right\}=\rho C p V \frac{d T}{d t}
$$

$\begin{array}{lll}\rho: & \text { Density of the node's medium } & {\left[\mathrm{kg} / \mathrm{m}^{3}\right]} \\ C p: & \text { Specific heat capacity of the node's medium } & {[\mathrm{J} / \mathrm{kg} \cdot \mathrm{K}]} \\ V: & \text { Volume of the node } & {\left[\mathrm{m}^{3}\right]} \\ T: & \text { Temperature of the node } & {\left[{ }^{\circ} \mathrm{C}\right]}\end{array}$

$\mathrm{t}: \quad$ Time [s]

172

173 The conduction term concerns the PCM nodes. It is given by the following equation:

174

175

176

177 Where:

$\mathrm{T}_{i}:$

Node temperature at node $\mathrm{i}$

$\mathrm{T}_{i-1}:$ :

Node temperature at node i-1

$\mathrm{T}_{i+1}$

Node temperature at node $i+1$

$R_{\text {cond }}=\frac{L / n}{S \cdot \lambda}$

Thermal resistance between neighbouring nodes

$L / n$ :

Length of node

Contact surface between neighbouring PCM nodes

Conductivity of the node's material $\left[{ }^{\circ} \mathrm{C}\right]$

$\left[{ }^{\circ} \mathrm{C}\right]$

$\left[{ }^{\circ} \mathrm{C}\right]$

[K/W]

[m]

$\left[\mathrm{m}^{2}\right]$

$[\mathrm{W} / \mathrm{m} \cdot \mathrm{K}]$ 
178 The advection term concerns the water layer and represents the heat transported into a water

179 node $\mathrm{i}$ from a neighbouring water node $\mathrm{i}-1$ and from the water node $\mathrm{i}$ to a neighbouring water

180 node $i+1$. In its explicit form, the advection term is given by the following equation:

181

182

183

184 Where

$\mathrm{T}_{i}:$

$\mathrm{T}_{i-1}:$ :

$\mathrm{T}_{i+1}$

$C p$ :

$\dot{\mathrm{m}}$ :

$$
\left\{\begin{array}{c}
\text { Advection } \\
\text { transfers } \\
\text { in node }
\end{array}\right\}=\dot{\mathrm{m}} C p\left(\mathrm{~T}_{j-1}-2 \cdot \mathrm{T}_{j}+\mathrm{T}_{j+1}\right)
$$

The convection term concerns the water layer and the most external nodes of the PCM layer, adjacent to the water layer. It is given by the following equation:

$$
\left\{\begin{array}{c}
\text { Convective } \\
\text { transfers } \\
\text { in node }
\end{array}\right\}=\frac{\left(\Delta T_{w-m}\right)}{R_{\text {conv }}}
$$

Where

$\Delta \mathrm{T}_{w-m}: \quad$ Temperature difference between adjacent water and PCM nodes

$R_{\text {conv }}=\frac{1}{S \cdot h} \quad$ Thermal resistance between neighbouring nodes

S :

Contact surface between water and PCM nodes the water (Equation 6) and PCM (Equation 7) nodes:

$$
\frac{\rho_{w} \cdot C p_{w} \cdot V_{w}}{d t} \cdot 2 \cdot\left(\overline{T_{w, J}}-T_{w, j}^{t-d t}\right)=\dot{\mathrm{m}} C p\left(\overline{T_{w, J-1}}-2 \overline{T_{w, J}}+\overline{T_{w, J+1}}\right)+\frac{\left(\overline{T_{m, J}}-\overline{T_{w, J}}\right)}{\left(R_{\text {cond }}+R_{\text {conv }}\right)}=
$$

198

$$
\begin{gathered}
\frac{\rho_{w} \cdot C p_{w} \cdot V_{w}}{d t} \cdot 2 \cdot\left(\overline{T_{w, J}}-T_{w, j}^{t-d t}\right)= \\
\dot{m} \cdot C p_{w} \cdot\left(\overline{T_{w, J-1}}-2 \overline{T_{w, J}}+\overline{T_{w, J+1}}\right)+\frac{\left(\overline{T_{m, J}}-\overline{T_{w, J}}\right)}{\left[\frac{1}{\left.\frac{\ln \frac{(m r-r)}{(m r-3 r / 2)}}{h \cdot 2 \cdot \pi \cdot(m-1) r \cdot(L / n)}+\frac{2 \pi \cdot k \cdot(L / n)}{2 \cdot}\right]}\right.}
\end{gathered}
$$




$$
\frac{\rho_{p} \cdot C p_{p} \cdot V_{p}}{d t} \cdot 2 \cdot\left(\overline{T_{l, j}}-T_{i, j}^{t-d t}\right)=\frac{\left(\overline{T_{l, j}-1}-2 \overline{T_{l, j}}+\overline{T_{1, l+1}}\right)}{R_{\text {cond }}}+\frac{\left(\overline{T_{l+1, j}}-\overline{T_{l, j}}\right)}{R_{\text {cond }}}+\frac{\left(\overline{T_{l-1, j}}-\overline{T_{l, j}}\right)}{R_{\text {cond }}}=
$$

$$
\frac{\rho_{p} \cdot C p_{p} \cdot V_{p}}{d t} \cdot 2 \cdot\left(\overline{T_{l, j}}-T_{i, j}^{t-d t}\right)=\frac{\left(\overline{T_{l, j+1}}-2 \overline{T_{l, j}}+\overline{T_{l, j}-1}\right)}{\frac{\mathrm{L}}{n \cdot \lambda \cdot \pi \cdot\left\{[(i-1) r]^{2}-[(i-2) r]^{2}\right\}}}+\frac{\left(\overline{T_{l+1, j}}-\overline{T_{l, j}}\right)}{\frac{\ln (i r-r / 2)}{(i r-3 r / 2)}}+\frac{\left(\overline{T_{l-1, j}}-\overline{T_{l, j}}\right)}{\frac{\ln \frac{(i r-3 r / 2)}{(i r-5 r / 2)}}{2 \pi \cdot \lambda \cdot(L / n)}}
$$

The average temperature

is introduced as the temperature variation is considered to be linear and the heat balance equation is formulated for each layer. The finite difference method is used to approximate the temperature derivative terms and the final form of the equations leads to a matrix formulation

214 for each layer [25].

\subsection{Model assumptions}

216 The objective of the numerical modelling was to reproduce the behaviour of the reference system in order to obtain a model able to calculate the temperature of the water after its heat exchange with the PCM modules. In order to develop an accurate and fast model, some assumptions had to be made.

220 First of all, the water flow is considered the same for all the sticks: it was calculated by

221 dividing the water flow that crosses each tank with the total number of gel sticks.

222 The model considers convection transfers between the water and the PCM layers, conduction transfers between the PCM layers and advection transfers between adjacent water nodes. It does not take into account convection inside the PCM during the melting and the liquid phases, irradiation and the thermal resistance of the enclosing plastic film of the gel sticks.

226 For the calculation of the temperature change rate in $\mathrm{j}=\mathrm{n}$ nodes a convection transfer was considered between PCM nodes and the average temperature of the adjacent water node at the previous time step $\left(\overline{T_{w, n}{ }^{t-d t}}\right)$. This was necessary as the water temperature value for the convection transfer calculation was needed but the temperature of the water node $\overline{T_{w, n}}$ could be obtained prior to calculation. The same assumption for the advection transfer calculation was used between the $\mathrm{j}=\mathrm{n}$ water node and the adjacent water node.

Another assumption concerned the $\mathrm{i}=1$ node: a unidirectional conduction transfer was considered from it to the $\mathrm{i}=2$ node (without considering an exchange between another internal node). This assumption was made for simplifying the calculation. In order to reduce the effect of this assumption, a fixed radius of $0.001 \mathrm{~m}$ (r1) to the $\mathrm{i}=1$ node was assigned. 
2.7 The heat transfer coefficient calculation

237 The convective heat transfer coefficient was calculated taking into account the packing 238 density of the gel sticks into the water $\operatorname{tanks}\left(\frac{D_{h}}{R_{c, \text { out }}}\right)$, with $D_{h}=$ equivalent diameter and $239 \mathrm{R}_{\mathrm{c}, \text { out }}=$ outer radius of the PCM cylinder. The relation between the packing density of the gel 240 sticks and the Nusselt number $(\mathrm{Nu})$ value is shown in figure 6:

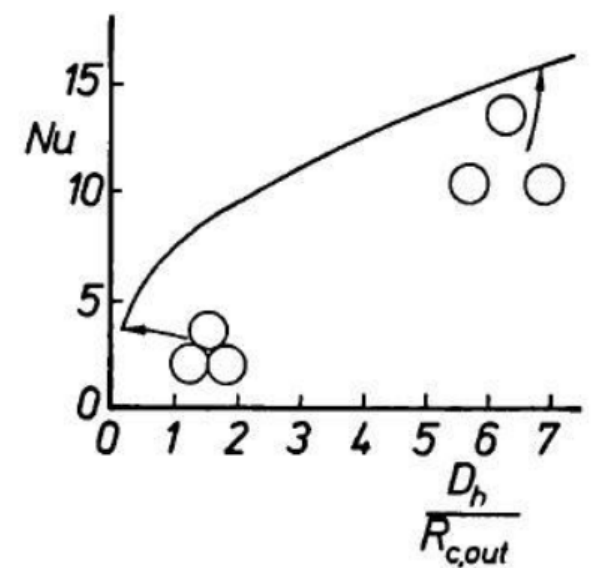

Figure 6 - Nusselt number values curve with reference to the packing density of the gel sticks for laminar flow [26].

251 Therefore, the Nusselt number can be defined using the formula [26]:

$$
N u=3.66+4.12 \cdot\left(\frac{D_{h}}{R_{c, \text { out }}}-0.205\right)^{0.569}
$$

Once calculated, the convective heat transfer coefficient can be calculated using the formula

$$
h=\frac{N u \cdot \lambda}{R_{c, \text { out }}}
$$

255 (with $\mathrm{R}_{\mathrm{c}, \text { out }}=$ external radius of the gel stick and $\lambda=$ water conductivity). 
Once the MATLAB Simulink model created, a simulation scenario was established, in order

258 to run the simulations. The scenario corresponds to a thermal cycle of the unit: charging and
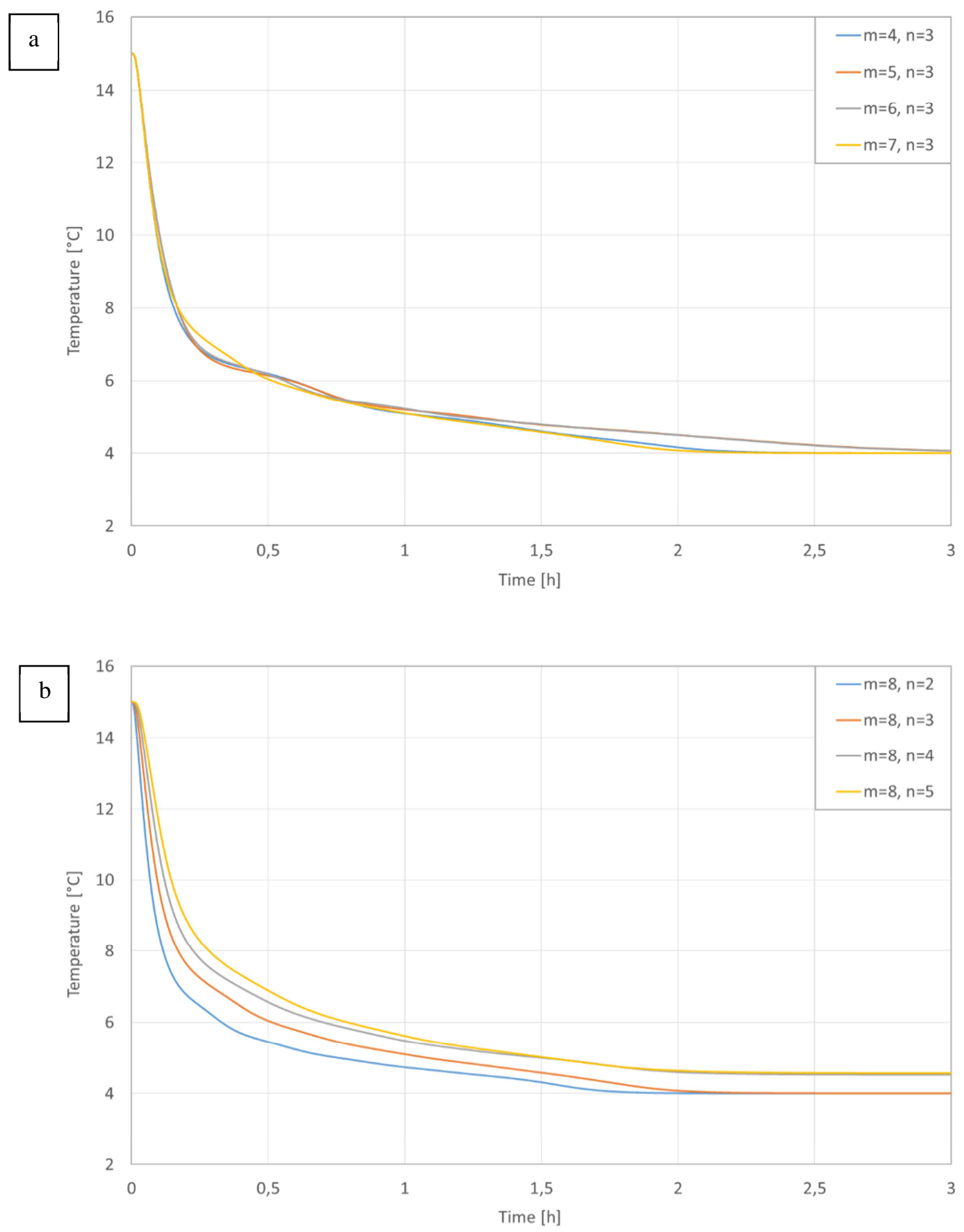

259 discharging period. It was decided to provide to the water-PCM stick exchanger a vertical

260 water flow rate of $0.3 \mathrm{~g} / \mathrm{s}$ (exactly the same water flow rate of the reference system for each 261 PCM stick) at $4{ }^{\circ} \mathrm{C}$ during 3 hours. The initial temperature of the exchanger was set at $15^{\circ} \mathrm{C}$ 262 and the acquisition time step at 10 seconds. The same scenario was adopted for each 263 simulation, changing only the nodes' discretization crosswise and lengthwise. An example of 264 the temperature evolution obtained for the same point through the comparison between models differently crosswise and lengthwise discretized is shown in figures 7.a and 7.b. 
The temperature curves of the figure 7.a correspond to the points indicated with red circles in figure 8.a, while those of the figure 7.b are indicated in figure 8.b.
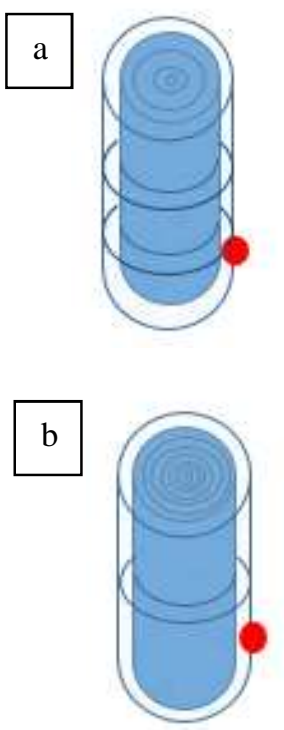
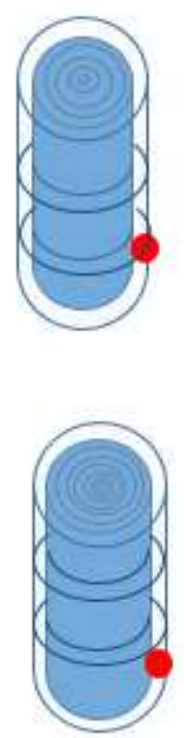
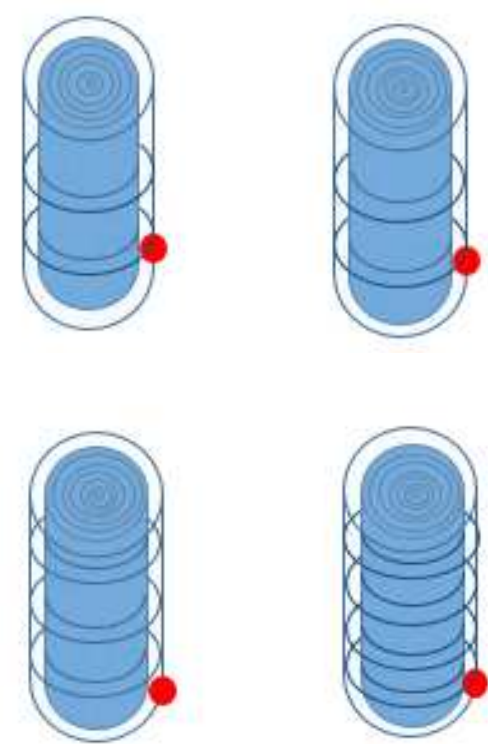

Figure 8-(a) Position of the points analyzed in figure 6.a in accordance to the different discretizations, (b) Position of the points analyzed in figure 6.b in accordance to the different discretizations.

As it can be noticed, every discretization caused a different temperature evolution curve in each node. This is due to the volume difference between all the different discretization configurations and, in the case shown in figure 6.b, to the different position of the analysed node in relation to the total length of the stick (as it can be observed in figure 8.b).

Once these first results obtained, a comparison was made between them and the in situ monitored values, in order to calibrate the model and validate it [27].

\section{NUMERICAL VALIDATION}

\subsection{The Computational Fluid Dynamics}

Once the model developed, a second numerical model was developed, using a different simulation method -the Computational Fluid Dynamics (CFD)-, in order to provide a first numerical validation, as the HIKARI's reference thermal storage system was still inoperative. As the objective of this work was to develop a fast and accurate numerical tool that could be used for the optimization of the reference heat-exchanger technology, this second model has been used only for validation purposes, because of its slow computational speed. For instance, for simulating the same phenomenon, the time needed is more than 100 times greater than the time needed using the MATLAB Simulink model.

304 The strategy of CFD is to replace the continuous problems domain with a discrete domain 305 using a cylindrical meshing method. The CFD works by means of mathematical modelling 306 (using the partial differential equations), numerical methods (discretisation and solution 307 techniques) and software tools (solvers, pre- and post-processing utilities). 
In this work, the software ANSYS Fluent was used. This software is based on the finite volume method. Navier-Stokes equations are solved numerically thanks to the discretisation of the domain into a finite set of control volumes and the sequent writing of the general conservation equations for mass, momentum, energy, species, etc. [28] All the partial differential equations are discretized into a system of algebraic equations and are solved numerically, in order to render the solution field.

\subsection{The CFD model development}

315 The created model should respect the geometry and the proprieties of the PCM water model developed through MATLAB Simulink. A water layer of $30 \mathrm{~cm}$ was nevertheless added on the top and on the bottom of the stick, in order to respect the geometry of the real system obtaining the CFD model represented in Figure 9. The meshing of the model was chosen using the meshing interface and the proprieties of the materials were added.

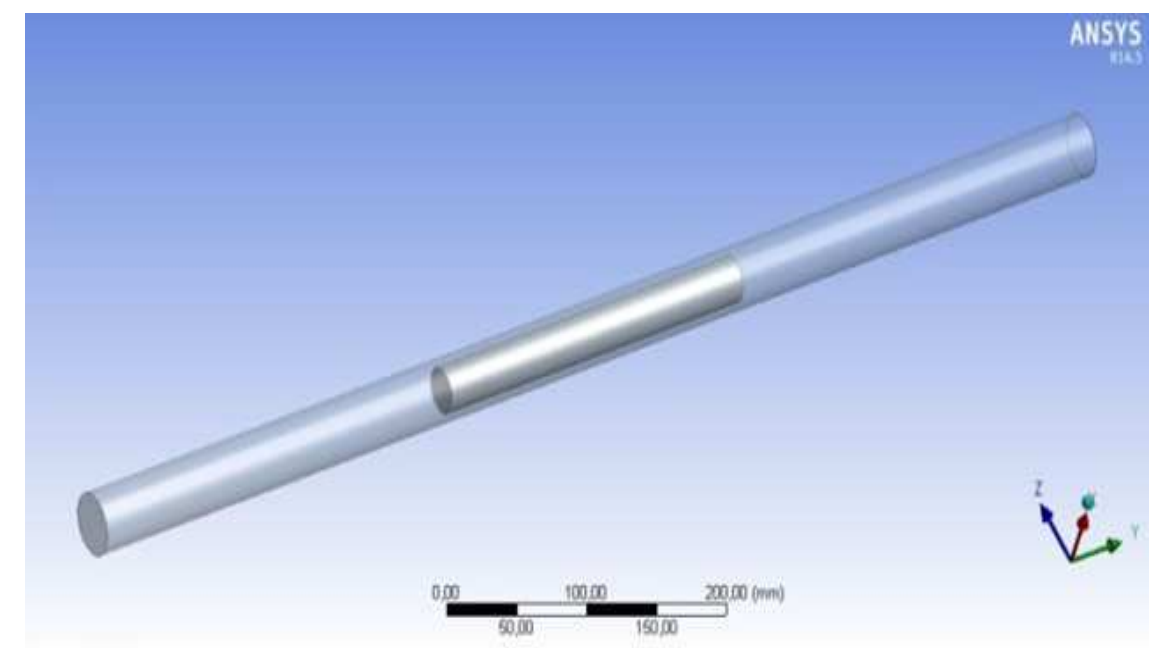

322 In order to make a valid comparison, we inserted the heat capacity values of the PCM obtained through the DSC method (as anticipated in paragraph 2.3).

324 Once the model and the materials properties defined, a simulation scenario was established, in 325 order to run the simulations.

326 The protocol is composed of 3 scenarios, with varying water flow rate $(0.15 \mathrm{~g} / \mathrm{s}, 0.3 \mathrm{~g} / \mathrm{s}$ and $0.45 \mathrm{~g} / \mathrm{s})$; every scenario consists of 2 steps:

1) The initial temperature of the exchanger is set at $12^{\circ} \mathrm{C}$ and the acquisition time step at 10 seconds. A water flow rate at $2^{\circ} \mathrm{C}$ is sent to the system during 330 minutes.

2) The initial temperature of the exchanger is set at $2^{\circ} \mathrm{C}$ and the acquisition time step at 10 seconds. A water flow rate at $12^{\circ} \mathrm{C}$ is sent to the system during 330 minutes. 
332 These water flow rates were chosen because $0.3 \mathrm{~g} / \mathrm{s}$ is the real flow rate that crosses a single 333 PCM stick in the HIKARI reference system, while $0.15 \mathrm{~g} / \mathrm{s}$ and $0.45 \mathrm{~g} / \mathrm{s}$ are respectively the 334 half and one and a half times its value.

$335 \quad 3.3$ Robustness and coherence test

336 Once all the simulations were run, we compared the temperature results obtained through the 337 developed MATLAB Simulink model and the ANSYS Fluent one for the same points. 338 Examples of the temperature evolution at the same point $(i=w, j=3$ ) for different 339 discretizations (see paragraph 2.4 for discretisation description) during the first and the 340 second step of the $0.45 \mathrm{~g} / \mathrm{s}$ water flow rate scenario are shown in figures 10.a and 10.b:
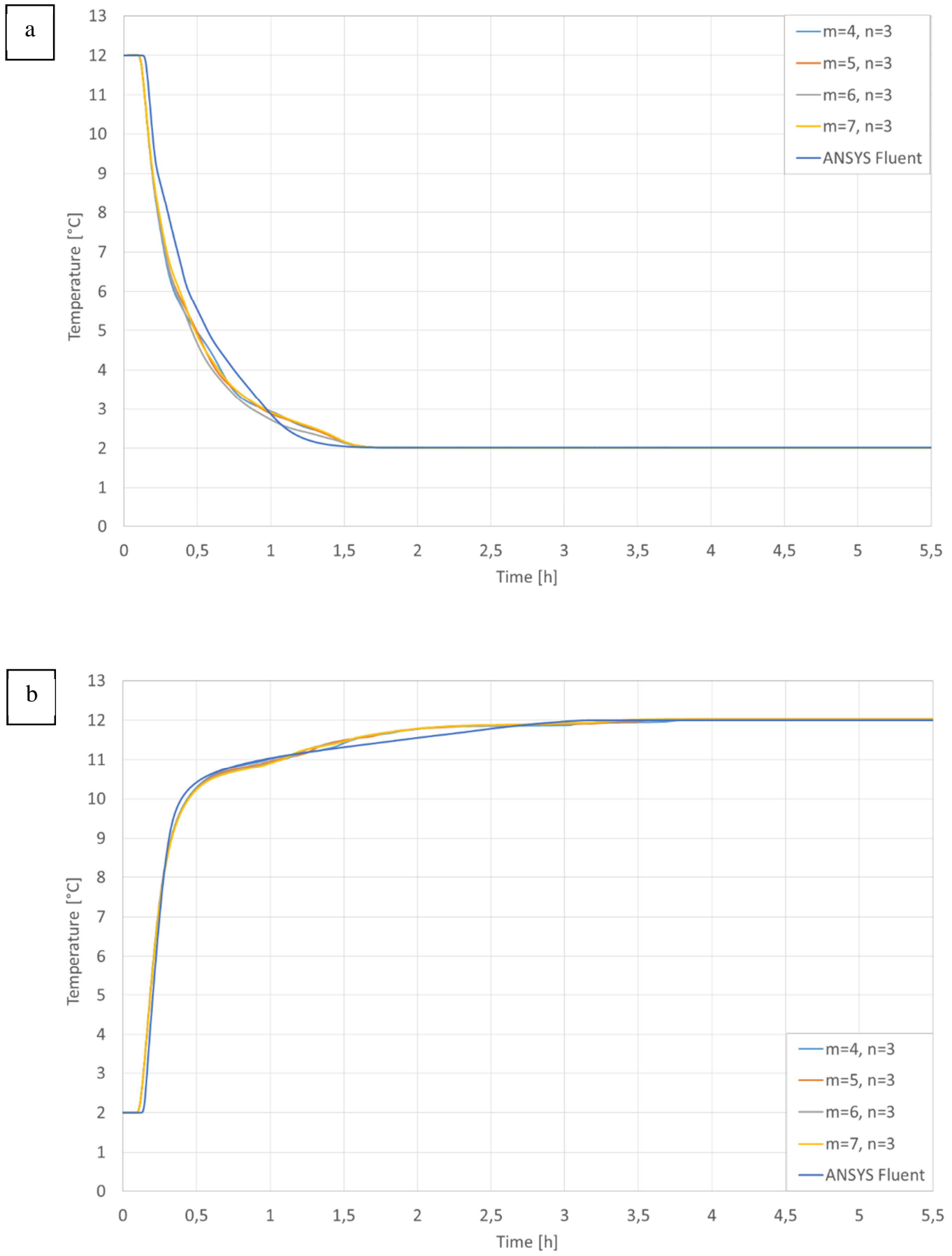

Figure 10 - Comparison of the temperature curves obtained with the two different software in the first step (a) and the second step (b) of the $0.45 \mathrm{~g} / \mathrm{s}$ water flow rate for the same point obtained using different crosswise discretization and the same lengthwise discretization $(n=3)$. 
The temperature evolution curves in figures 10.a and 10.b show the values obtained through different crosswise discretisation for the same water node $(i=w, j=3)$ shown in figure 8.a, that corresponds to the point shown in figure 11 in the ANSYS Fluent model:

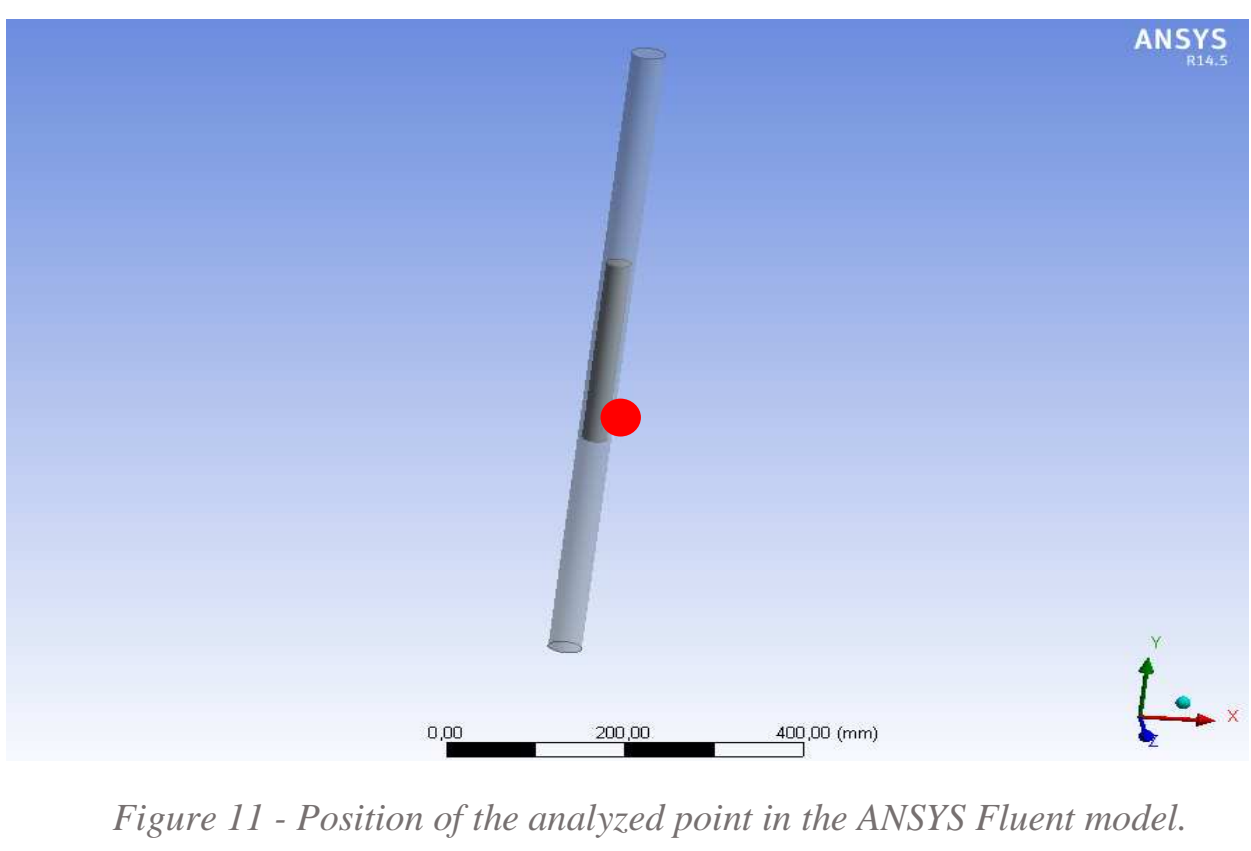

A small time difference between the two software is noticed, this concerning the point when the water starts being chilled (or heated). In fact, for all the scenarios the ANSYS Fluent model starts being chilled (and heated) some minutes after the MATLAB Simulink one, with a difference of the order of 5 minutes. This time delay could be due to the difference between the dimension of the meshing in the two software and to the different solving methods. Another interesting phenomenon that can be observed is that the MATLAB Simulink model provides some not acceptable results according to physics when it is lengthwise discretized in 4 or more nodes. In fact, one of the assumption made during the modelling phase was that the PCM-water system was considered adiabatic, as all the tanks of the reference system were thermally insulated. For this reason, if a continue water flow rate at $2^{\circ} \mathrm{C}$ is sent to the system, the whole system should stabilise at $2^{\circ} \mathrm{C}$ after some time, as there is no heat exchange with the external ambient environment. Instead, some of the results obtained through particular discretizations, never reach the temperature of $2{ }^{\circ} \mathrm{C}$. This could be due to a particular relationship between the length and the thickness of the nodes that prevents the software adequately from solving the heat balance equations for those discretizations.

\subsection{Numerical calibration}

379 Following the different comparisons between the ANSYS Fluent model and the MATLAB

380 Simulink model's results for the same node, an error analysis between the results obtained 381 through the two software was carried out.

382 In order to test the accuracy of the MATLAB Model two criteria were calculated: 
- $\quad$ The Normalized Mean Bias Error (NMBE)

$$
N M B E=\frac{\sum_{i=1}^{n}\left(y_{\text {simulated }, i}-y_{\text {reference }, i}\right)}{\bar{y}_{\text {reference }} \times(n-p)} \times 100
$$

- The Coefficient of Variation of the Root Mean Squared Error (CV(RMSE))

$$
C V(R M S E)=\frac{1}{\bar{y}_{\text {reference }}} \times \sqrt{\frac{\sum_{i=1}^{n}\left(y_{\text {simulated }, i}-y_{\text {reference }, i}\right)^{2}}{(n-p-1)}} \times 100
$$

392 Typically and according to ASHRAE, models are declared to be calibrated if they produce

393 NMBEs within $\pm 10 \%$ and CV(RMSE)s within $\pm 30 \%$ [29].

394 For the calculation of these coefficients, the ANSYS Fluent model results were considered as

395 the "reference values", and the difference between them and the MATLAB Simulink model 396 results was calculated.

397 It was noticed that the discretization that provided the best results was the one with 8 nodes 398 crosswise ( 7 concentric cylinder and a water layer) and 3 nodes lengthwise $(m=7, n=3)$.

399 In Table 1 the NMBE and the CV(RMSE) values for this discretization are showed, while 400 Figures 12.a, 12.b and 12.c illustrate the difference between the results obtained with the two 401 software for the point $(\mathrm{i}=\mathrm{w}, \mathrm{j}=3$ ) when the flow rates is $0.15 \mathrm{~g} / \mathrm{s}, 0.3 \mathrm{~g} / \mathrm{s}$ and $0.45 \mathrm{~g} / \mathrm{s}$. 


\begin{tabular}{|c|c|c|}
\hline Scenario & Statistical Index & Values [\%] \\
\hline \multirow{2}{*}{ 1) Flow rate $: 0.15 \mathrm{~g} / \mathrm{s}$} & NMBE & -0.26 \\
\cline { 2 - 3 } & CV(RMSE) & 17 \\
\hline \multirow{2}{*}{ 2) Flow rate $: 0.3 \mathrm{~g} / \mathrm{s}$} & NMBE & -0.18 \\
\cline { 2 - 3 } & CV(RMSE) & 12 \\
\hline \multirow{2}{*}{ 3) Flow rate $: 0.45 \mathrm{~g} / \mathrm{s}$} & NMBE & -0.21 \\
\cline { 2 - 3 } & CV(RMSE) & 13 \\
\hline
\end{tabular}



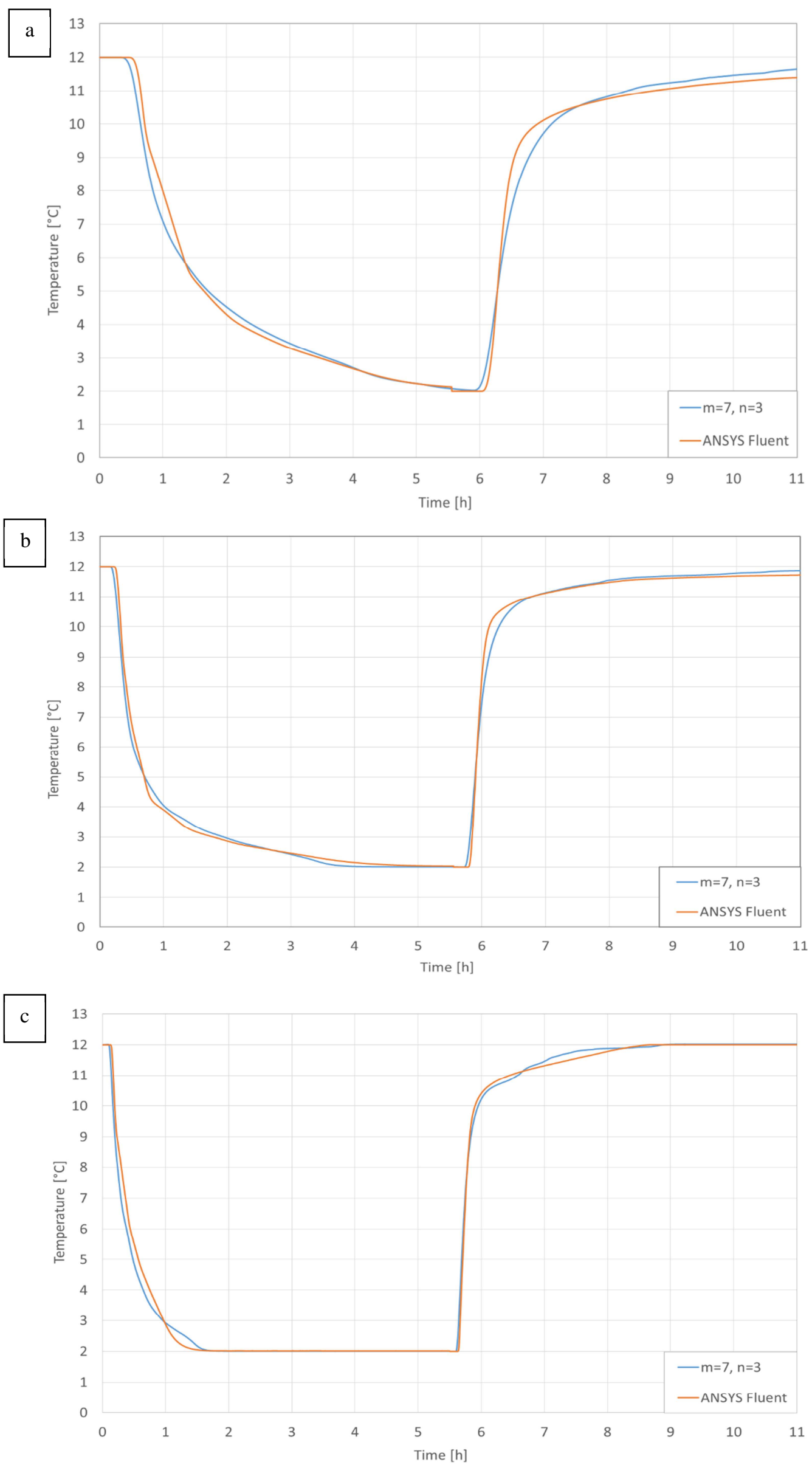
The behaviour of the two models is similar, even though some discretization provided a time

408 difference between the two software's results. For this reason, it was necessary to conduct an 409 experimental validation in order to obtain a clearer view on eventual inaccurate assumptions 410 or input errors.

\section{EXPERIMENTAL VALIDATION}

412 An original experimental prototype reproducing the HIKARI cold storage system was 413 developed in the ENTPE Laboratory of Tribology and Systems Dynamics (LTDS), in order to 414 perform a first experimental validation. An extended description of the prototype can be 415 found in Reference [30].

\section{$416 \quad 4.1$ The experimental prototype}

417 The prototype (Figure 13.a) consists of two insulated Plexiglas tanks filled with water, into 418 one of which is inserted a plastic case filled with the PCM sticks. The temperature of the 419 water on the tank 1 is regulated by an external cryostat and then it is directed to tank 2 , where 420 it crosses the PCM modules layer, charging and discharging it.

421 The scheme of the prototype is showed in Figure 13.b:
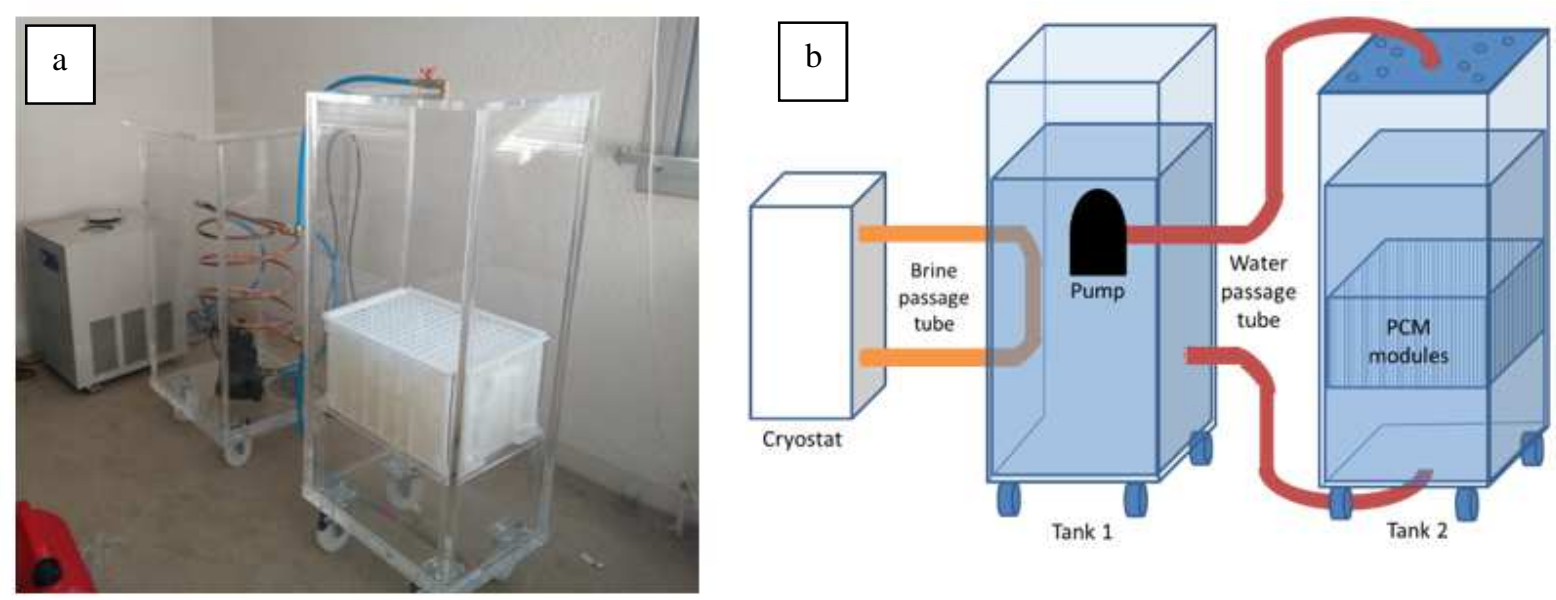

Figure $13-(a)$ The experimental prototype and (b) scheme of the experimental prototype.

At first, only one PCM module has been inserted in tank 2, in order to test the PCM-water heat placed at 15 points of interest as illustrated in Figure 14.b. 

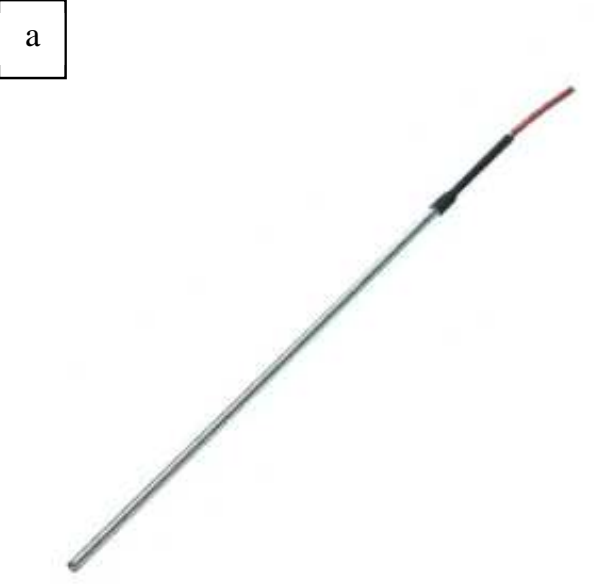

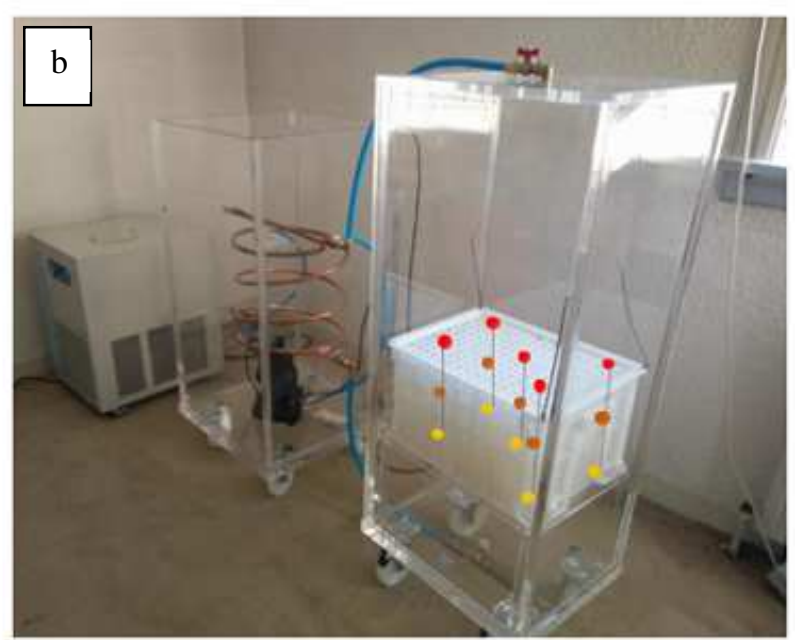

Figure 14 - (a) PT100 temperature sensor and (b) positions of the 15 points analyzed using the temperature sensors.

433 In a second step, another PCM module has been inserted on top of the first one in tank 2, in 434 order to test if the model is able to simulate the heat exchange module-module beside the 435 module-water flow one. In this second case the PT100 temperature sensors have been placed 436 at 15 different points corresponding to the points analyzed in the model of a double PCM gel 437 stick-water heat exchange (scheme in Figure 15).

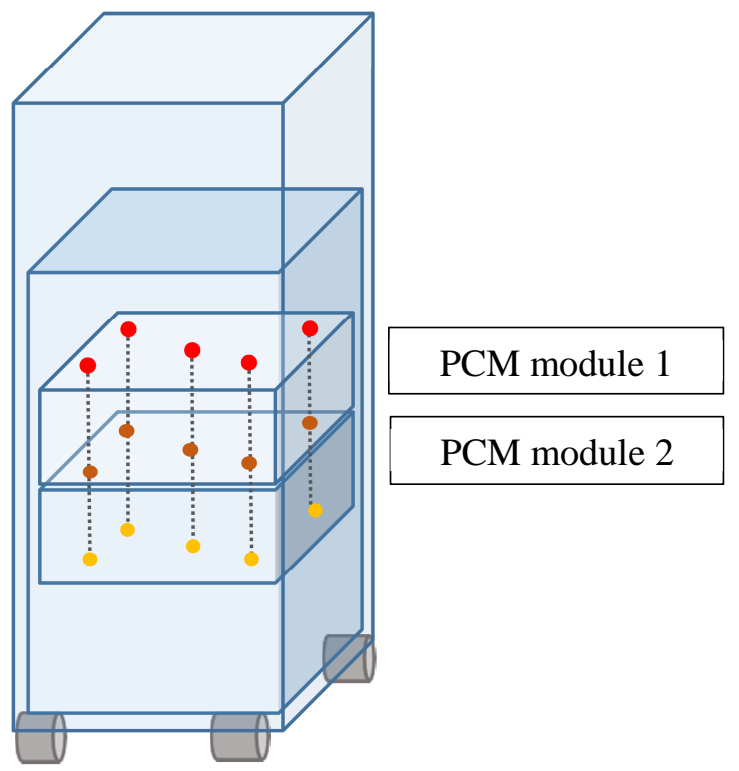

Figure 15 - Positions of the 15 points analyzed using the temperature sensors when 2 PCM modules are inserted in tank 2.

440 An experimental protocol was established in order to test the "goodness of fit" between the 441 numerical model prediction and the experimental data in different scenarios. The objective of 442 this protocol was to recreate particular situations that could be registered in different systems 
operating scenarios. In particular, the objective was to overcome two factors that were imposed by the real functioning of the reference system: the constant water flow rate and temperature range.

The water flow rates were chosen considering the flow rate for every plastic case of the reference system during the charge period $(50 \mathrm{~g} / \mathrm{s})$.

It was decided to test the heat exchange when the flow rate between the two tanks is the half $(25 \mathrm{~g} / \mathrm{s})$ and the double $(100 \mathrm{~g} / \mathrm{s})$ of that value in order to verify the validity of the numerical model when it is used in different contexts. As the reference storage system works between 6 and $10^{\circ} \mathrm{C}$, it was decided to operate the prototype with water temperatures ranging from 3 to $20^{\circ} \mathrm{C}$. At the end, the protocol was composed of 6 scenarios, with varying water flow rate $(25$, 50 and $100 \mathrm{~g} / \mathrm{s}$ ) and different PCM quantity (1 or 2 plastic cases).

\section{Every scenario consisted of 4 steps:}

1) In the first step the cryostat was set to $-2{ }^{\circ} \mathrm{C}$. As the pump that regulated the water flow between the two tanks was off, only the water of tank 1 was chilled.

2) After 8 hours, the pump was switched on, so the heat exchange between the PCM modules and the chilled water flow coming from the tank 1 happened, reproducing the "charging" phase of the reference system.

3) After 16 hours, the pump that regulated the water flow between the two tanks was switched off and the cryostat was set to $20^{\circ} \mathrm{C}$.

4) Finally, after 8 hours, the pump was switched on again, so the heat exchange between the PCM modules and the heated water flow occurred, reproducing the "discharging" phase of the reference system during $16 \mathrm{~h}$.

For every scenario, the temperature evolution was registered.

\subsection{Experimental validation}

The results were grouped in 3 outputs, in order to reduce the effect of the proximity of the sensors at the tank walls:

1) Inlet (Arithmetical mean of the temperatures registered by the 5 sensors placed at the top of the plastic cases)

2) Intermediate (Arithmetical mean of the temperatures registered by the 5 sensors placed in the middle of the plastic cases)

3) Outlet (Arithmetical mean of the temperatures registered by the 5 sensors placed at the bottom of the plastic cases).

Using these pooled data it will be possible to validate the MATLAB-Simulink model comparing the water temperature evolution along the stick length during the heat exchange.

The output that was compared to the results obtained through the numerical model was the "outlet" one, as this would be one of the key parameters in the optimization phase of this 
technology. Two examples of comparison between the prototype data and the model outlet

481 results are shown in figures 16 .a and 16.b:
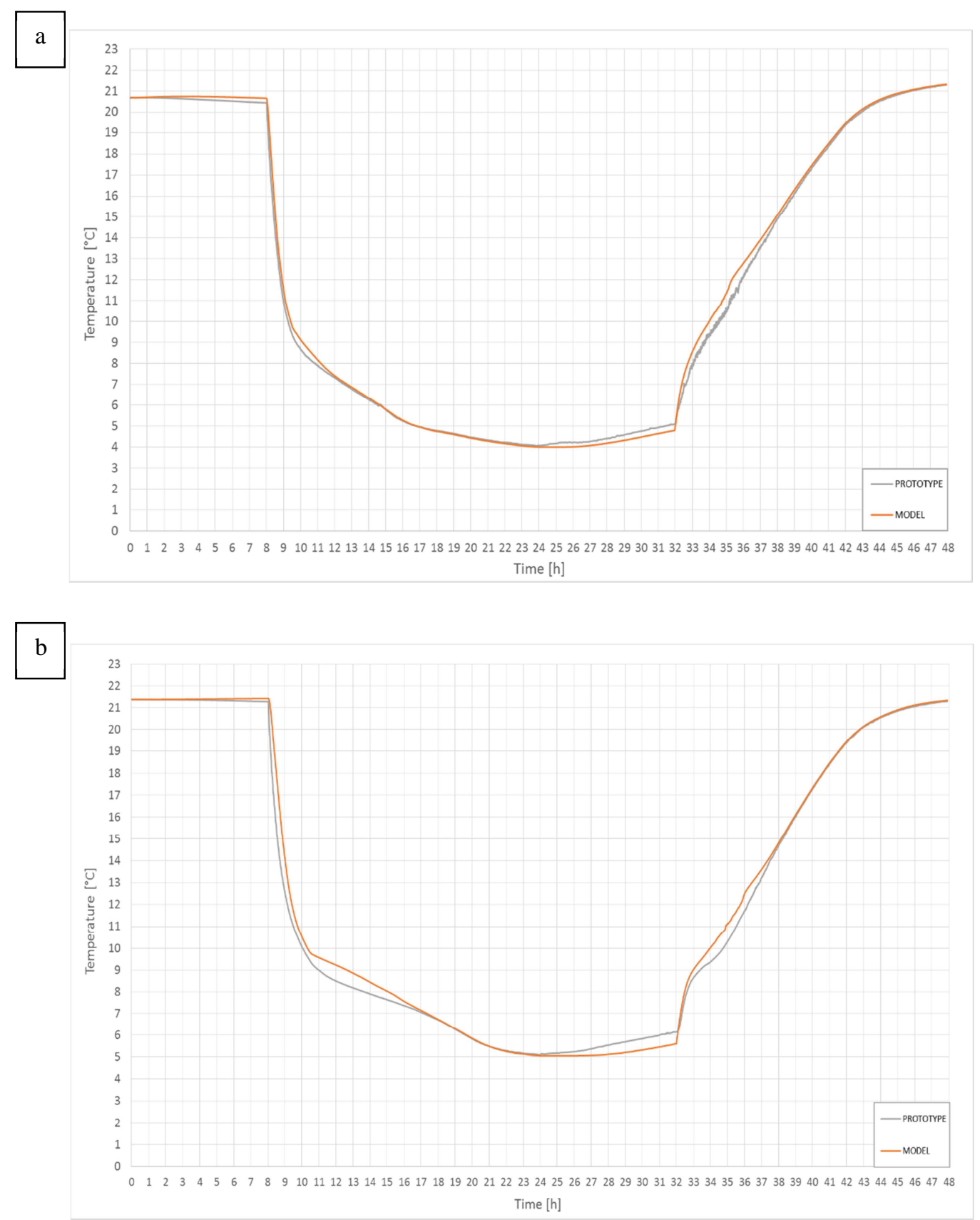
in tank 2, (b) two PCM modules are inserted in tank 2.

485 As in the numerical calibration case, the Normalized Mean Bias Error (NMBE) and the 486 Coefficient of Variation of the Root Mean Squared Error (CV(RMSE)) were used as validation criteria, considering the prototype data as the reference values.

488 The results for each scenario are shown in table 2: 

the prototype monitoring and the discretization $(m=7, n=3)$ of the MATLAB Simulink model.

\begin{tabular}{|c|c|c|}
\hline Scenario & Statistical Index & Values [\%] \\
\hline 1) 1 Plastic case & NMBE & 1,07 \\
\cline { 2 - 3 } Flow rate $: 25 \mathrm{~g} / \mathrm{s}$ & CV(RMSE) & 3,21 \\
\hline 2) 1 Plastic case & NMBE & 0,97 \\
\cline { 2 - 3 } Flow rate $: 50 \mathrm{~g} / \mathrm{s}$ & CV(RMSE) & 2,57 \\
\hline 3) 1 Plastic case & NMBE & 0,56 \\
\cline { 2 - 3 } Flow rate $: 100 \mathrm{~g} / \mathrm{s}$ & CV(RMSE) & 2,72 \\
\hline \multirow{2}{*}{ 4) 2 Plastic cases } & NMBE & 2,08 \\
\cline { 2 - 3 } Flow rate $: 25 \mathrm{~g} / \mathrm{s}$ & CV(RMSE) & 5,79 \\
\hline \multirow{2}{*}{ 5) 2 Plastic cases } & NMBE & 1,23 \\
\hline Flow rate $: 50 \mathrm{~g} / \mathrm{s}$ & CV(RMSE) & 3,72 \\
\hline \multirow{2}{*}{ 6) 2 Plastic cases } & NMBE & $-0,05$ \\
\hline Flow rate $: 100 \mathrm{~g} / \mathrm{s}$ & CV(RMSE) & 2,81 \\
\cline { 2 - 3 }
\end{tabular}

492

493

The validation results showed that the numerical model produces the same behaviour as the prototype one. The Normalized Mean Bias Error (NMBE) and the Coefficient of Variation of the Root Mean Squared Error (CV(RMSE)) used to analyse the validation results showed that the reference model was very accurate and fitted largely the ASHRAE specifications for all tested configurations.

\section{IN SITU VALIDATION}

Once the HIKARI buildings inaugurated, the monitoring process started in order to evaluate the performance of the building and its equipment, so as to reach the positive energy balance target. In order to test the HIKARI energy behaviour, all the measured data are compared every month with the expected simulated data for the same weather conditions to detect any failure or decay between actual and planned performance of equipment and systems.

The temperature values registered during the cold storage system monitoring campaign were compared to those obtained using the numerical model presented in this work in order to make an in situ validation. 
510 In the specific case of the HIKARI's cold storage system, 14 temperature sensors were 511 inserted in each insulated tank of the system in 14 points of interest represented in the section 512 of the tank 1 shown in Figure 17 and the scheme shown in Figure 18.

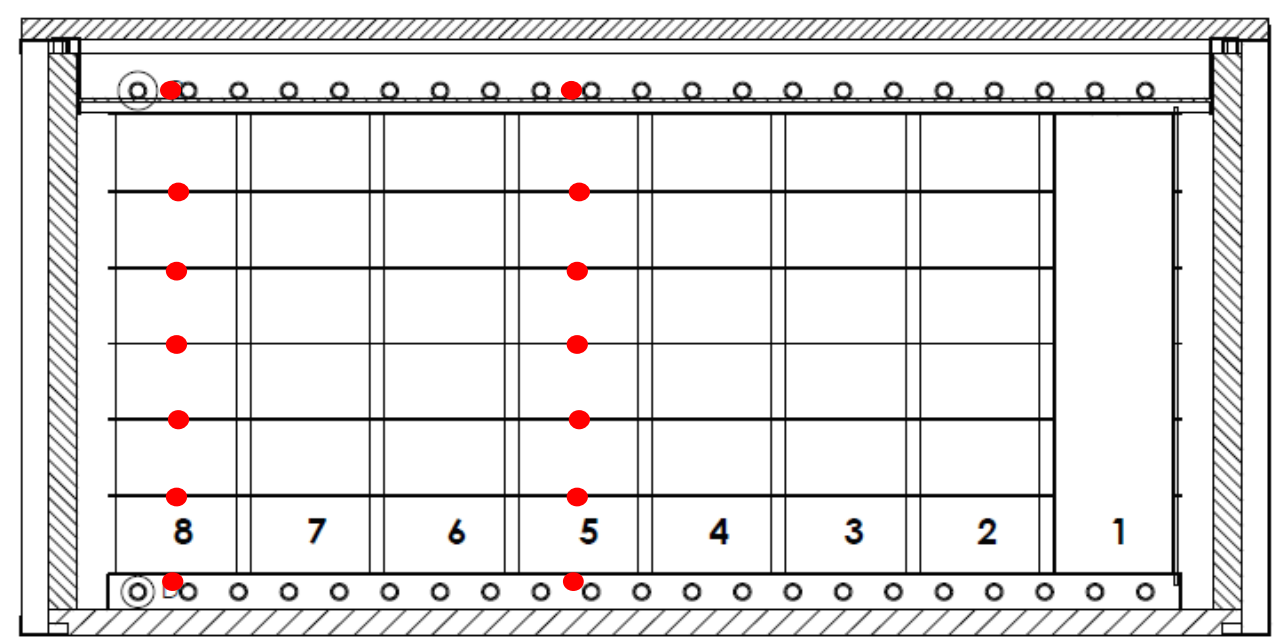

Figure 17 - Section of the tank 1 showing the positions of the temperature sensors.

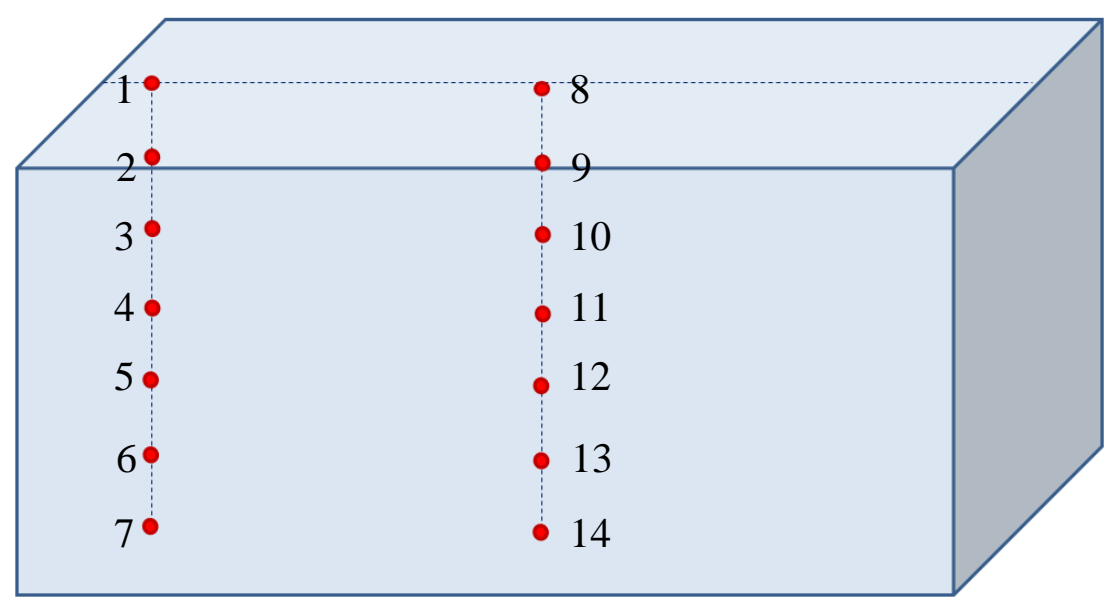

Figure 18 - Scheme of the positions of the temperature sensors in each

515 As it can be noticed, the temperature sensors were inserted at the top and the bottom of the set 516 of plastic cases filled with the PCM modules and between all the plastic cases. The central 517 and an external column of plastic cases were chosen in order to test the impact of the external 518 leakage and the homogeneity of the vertical heat transfer. The temperature values were 519 registered with a time step of 60 seconds.

520 An example of the temperature recorded for the tank 1 by the 14 sensors is shown in Figure 521 19: 


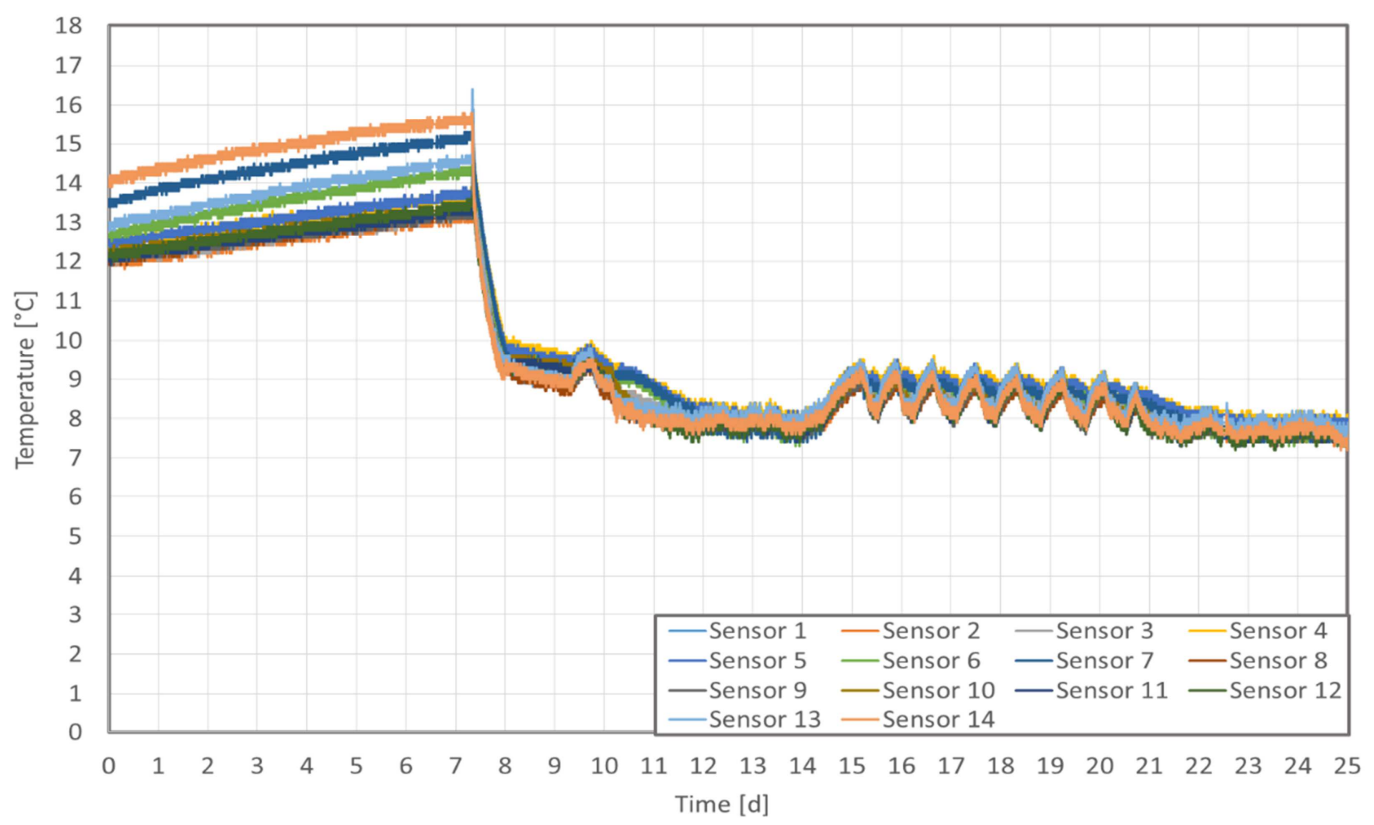

Figure 19- Example of the temperature recorded by the 14 sensors of the tank 1.

$522 \quad 5.2$ In situ validation

523 A comparison was then made between the results obtained through the model and the in situ

524 data, as registered by the last sensor of the central column of the tank (number 14 as shown in 525 Figure 19), corresponding to the output value of our model.

526 As for the numerical calibration (Paragraph 3.4), the Normalized Mean Bias Error (NMBE)

527 and the Coefficient of Variation of the Root Mean Squared Error (CV(RMSE)) between the 528 reference results (the data obtained through the in situ monitoring) and the model results were 529 calculated. The graphic showing the difference between the in situ data recorded by the sensor 53014 and the model results for this discretization is shown in Figure 20, while the results of the 531 NMBE and CV(RMSE) criteria are shown in table 3:

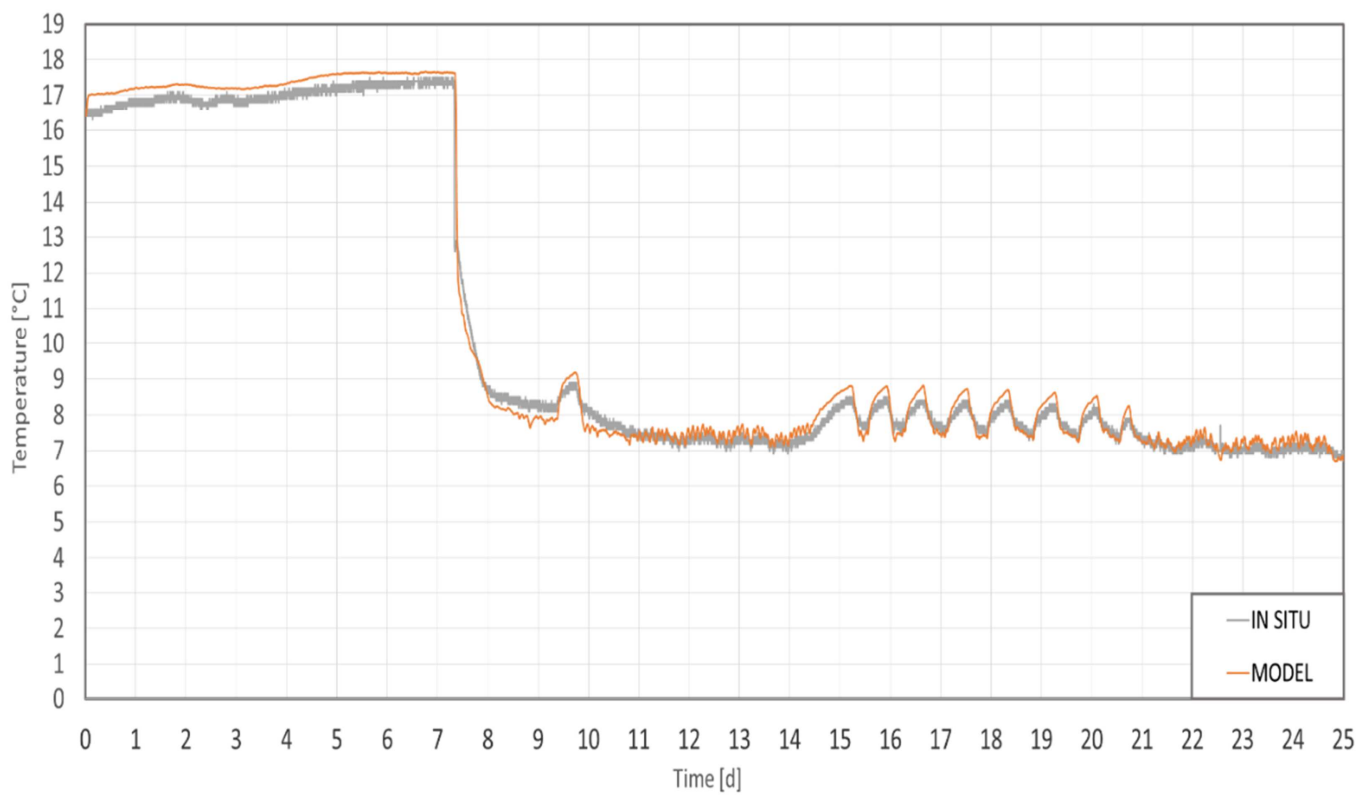

Figure 20 - Comparison of the temperature curves recorded by sensor 14 and the model results. 


\begin{tabular}{|l|l|}
\hline Normalized Mean Bias Error & $1,86 \%$ \\
\hline Coefficient of Variance of Root Mean Square Error & $4,02 \%$ \\
\hline
\end{tabular}

533

534

535

For this case as well, the obtained results show that the MATLAB-Simulink model is able to reproduce the energy behaviour of the reference thermal energy storage system at low temperatures.

\section{CONCLUSIONS}

The results obtained show that the choice of the heat balance approach provided a fast, accurate and re-utilizable numerical model able to reproduce the heat exchange between the Phase Change Material (PCM) contained in the reference system and a water flow, that can be used for the optimization of this energy storage technology.

Once developed, the model was firstly calibrated using the results obtained through a second numerical model that was developed using the Computational Fluid Dynamics method. The results of this calibration showed that the model was accurate and faster in long duration simulation studies when compared to ANSYS Fluent results.

A first experimental validation was realized using an original experimental prototype reproducing the HIKARI's cold storage system, which was designed and developed in order to obtain reliable experimental data useful for a complete experimental validation of the numerical model and for the validation of other kinds of phase change materials in the future. For this reason, the prototype had the advantage to be easy to dissemble and reassemble (in case of transport) but at the same time able to provide fast heat transfer rates to the PCM and to record all the temperature changes with a very good precision.

Finally, a second experimental validation was realized once the HIKARI in situ data obtained thanks to in-situ monitoring. This comparison allowed us to make the in situ validation of the model.

The results obtained through its construction showed that the developed model was able to reproduce the PCM-water heat exchange with a high accuracy. A slightly delayed response during its phase change can be noticed (maybe due to the effect of further minor heat transfer phenomena that were not taken into account in the heat balance equations definition). Nevertheless, the use of the Normalized Mean Bias Error (NMBE) and the Coefficient of Variation of the Root Mean Squared Error (CV(RMSE)), showed that the developed model was very accurate and fitted largely the ASHRAE specifications for all tested configurations. 
Once numerically calibrated and experimentally validated, the model was coupled to the HIKARI's HVAC systems model in order to test the effects of geometry and thermo-physical changes of the reference system on the efficiency of some other systems and on HIKARI energy needs.

Applying the validation techniques shown in this work, new geometries and solving methods can be tested as well for this and other systems in the future.

[1] IEA-ETSAP and IRENA (2013) “Thermal Energy Storage. Technology Brief.”

[2] Stathopoulos N., El Mankibi M., Issoglio R., Michel P., Haghighat H. (2016) “Air-PCM heat exchanger for peak load management: Experimental and simulation." Solar Energy. Vol. 132, pp 453-466.

[3] Alva G., Liu L., Huang X., Fang G. (2017) "Thermal energy storage materials and systems for solar energy applications”. Renewable and Sustainable Energy Reviews. Vol. 68, Part 1, pp 693-706.

[4] Reddy K.S., Mudgal V., Mallick T.K. (2018) "Review of latent heat thermal energy storage for improved material stability and effective load management". Journal of Energy Storage. Vol. 15, pp 205-227.

[5] Zalba B., Marín J. M., Cabeza L. F., Mehling H. (2003)” Review on thermal energy storage with phase change: materials, heat transfer analysis and applications". Applied Thermal Engineering. Vol. 23, pp 251-283.

[6] Khudhair A.M., Farid M.M. (2004) "A review on energy conservation in building applications with thermal storage by latent heat using phase change materials". Energy Conversion and Management. Vol. 45 pp 263-275.

[7] Zhu N., Ma Z., Wang S. (2009) "Dynamic characteristics and energy performance of buildings using phase change materials: A review". Energy Conversion and Management.Vol.50, Issue 12, pp 3169-3181.

[8] Cabeza L. F., Castell A., Barreneche C., de Gracia A., Fernández A. I. (2011) "Materials used as PCM in thermal energy storage in buildings: A review". Renewable and Sustainable Energy Reviews, Vol. 15, pp 1675-1695.

[9] Roccamena L. (2017) "Optimization of an innovative thermal energy storage technology at low temperatures when coupled to multi-source energy architectures". Doctoral thesis. Ecole Nationale des Travaux Publics de l'Etat, Lyon (France).

[10] Simon F., Corgier D., El Mankibi M. "Innovative and energy-flexible design for a positive energy balance plot in Lyon (France): the HIKARI project". Nordic Passive House Conference, 27-29 September 2017, Helsinki (Finland).

[11] Souayfane F., Fardoun F., Biwole P.-H. (2016) "Phase change materials (PCM) for cooling applications in buildings: A review”. Energy and Buildings, Vol. 129, pp 396-431. 
600

601

602

603

604

605

606

607

608

609

610

611

612

613

614

615

616

617

618

619

620

621

622

623

624

625

626

627

628

629

630

631

632

633

634

635

[12] Chaiyat N. (2015) "Energy and economic analysis of a building air-conditioner with a phase change material (PCM)". Energy Conversion and Management, Vol.94, pp 150-158.

[13] Fang G., Wu S., Liu X. (2010) "Experimental study on cool storage air-conditioning system with spherical capsules packed bed”. Energy and Buildings, Vol. 42, pp 1056-1062.

[14] Kasaeian A., Bahrami L., Pourfayaz F., Khodabandeh E., Yan W. (2017) "Experimental Studies on the Applications of PCMs and Nano-PCMs in Buildings: A Critical Review", Energy and Buildings, Vol. 154, pp 96-112.

[15] Liu S., Li Y., Zhang Y. (2014) "Mathematical solutions and numerical models employed for the investigations of PCMs' phase transformations". Renewable and Sustainable Energy Reviews. Vol. 33, pp 659-674.

[16] Radhakrishnan K.B., Balakrishnan A.R. (1992) "Heat transfer analysis of thermal energy storage using phase change materials". Heat Recovery Systems and CHP. Vol. 12, Issue 5, pp 427-435.

[17] Kürklü A., Wheldon A., Hadley P. (1996) "Mathematical modeling of the thermal performance of a phase-change material store: cooling cycle". Applied Thermal Engineering. Vol. 16, Issue 7, pp 613-623.

[18] El Mankibi M., Stathopoulos N., Rezaï N., Zoubir A. (2015) "Optimization of an AirPCM heat exchanger and elaboration of peak power reduction strategies". Energy and Buildings, Vol. 106, pp 74-86.

[19] Liu Z., Yu Z., Yang T., Roccamena L., Sun P., Li S., Zhang G., El Mankibi M. (2019), "Numerical modeling and parametric study of a vertical earth-to-air heat exchanger system”. Energy, Vol.172, pp 220-231.

[20] Van Schijndel A.W.M. (2009) "Integrated modeling of dynamic heat, air and moisture processes in buildings and systems using SimuLink and COMSOL". Building Simulation. Vol. 2, Issue 2, pp 143-155.

[21] AL-Saadi S.N., Zhai Z. (2013) "Modeling phase change materials embedded in building enclosure: A review”. Renewable and Sustainable Energy Reviews. Vol. 21, pp 659-673.

[22] Bouhal T., El Rhafiki T., Kousksou T., Jamil A., Zeraouli Y. (2018) "PCM addition inside solar water heaters: Numerical comparative approach". Journal of Energy Storage. Vol.19, pp 232-246.

[23] Idelsohn S.R., Storti M.A., Crivelli L.A. (1994) "Numerical methods in phase-change problems". Archives of Computational Methods in Engineering. Vol. 1, Issue 1, pp 4974.

[24] Hu H., Argyropoulos S.A. (1996) "Mathematical modelling of solidification and melting: a review". Modelling and Simulation in Materials Science and Engineering. Vol.4, pp. 371-396. 
636 [25] Stathopoulos N. (2015) "Optimisation numérique et expérimentale de stratégies 637 d'effacement énergétique”. Doctoral thesis. Ecole Nationale des Travaux Publics de 638 l'Etat, Lyon (France).

639 [26] Visser H. (1986) "Energy storage in phase-change materials. Development of a 640 component model compatible whit the "TRNSYS" transient simulation program". 641 Commission of European communities.

642 [27] Dutil Y., Rousse D.R., Ben Salah N., Lassue S., Zalewski L. (2011) “A review on phase643 change materials: Mathematical modeling and simulations". Renewable and Sustainable 644 Energy Reviews. Vol.15, Issue 1, pp 112-130.

645 [28] Fan Y., Ito K. (2014) “Optimization of indoor environmental quality and ventilation load 646 in office space by multilevel coupling of building energy simulation and computational 647 fluid dynamics”. Building Simulation. Vol. 7, Issue 6, pp 649-659.

648 [29] ASHRAE (2002) Guideline 14 - Measurement of Energy and Demand Savings.

649 [30] Roccamena L., El Mankibi M., Stathopoulos N. (2018) "Experimental test bed design 650 and development for PCM-water exchangers characterization". Sustainable Cities and $651 \quad$ Society. Vol. 37, pp 241-249. 